\title{
Genetic characterization of Fusarium graminearum and $F$. culmorum isolates from Turkey by using random-amplified polymorphic DNA
}

\author{
E. Yörük ${ }^{1}$ and G. Albayrak ${ }^{2}$ \\ ${ }^{1}$ Programme of Molecular Biology and Genetics, Institute of Science, \\ Istanbul University, Istanbul, Turkey \\ ${ }^{2}$ Department of Molecular Biology and Genetics, Faculty of Science, \\ Istanbul University, Istanbul, Turkey \\ Corresponding author: G. Albayrak \\ E-mail: gulruh@istanbul.edu.tr
}

Genet. Mol. Res. 12 (2): 1360-1372 (2013)

Received August 7, 2012

Accepted January 27, 2013

Published April 25, 2013

DOI http://dx.doi.org/10.4238/2013.April.25.7

\begin{abstract}
Five Fusarium graminearum and 12 F. culmorum isolates, primarily pathogenic species of Fusarium head blight, were obtained from naturally infected wheat from various agro-ecological regions of Turkey. Genotyping of the isolates was carried out using random-amplified polymorphic DNA (RAPD). Sixty-five 10-mer oligonucleotide primers were used to amplify the RAPD markers. Among them, 50 primers produced strong and reproducible DNA amplicons. The remaining primers generated either insufficient or no amplification patterns. In total, 1200 fragments were scored, 311 of which were determined to be polymorphic and unique to the isolates. The produced RAPD markers ranged from 0.2 to $5 \mathrm{~kb}$. The mean genetic similarity values of the $F$. graminearum and $F$. culmorum isolates were 61.5 and $65 \%$, respectively. The similarity coefficient was 43 to $76.1 \%$ among $F$. graminearum isolates and 49 to $81.1 \%$ among $F$. culmorum isolates. Genetically, the most similar $F$. graminearum isolates were F6 and F7 (76.1\%), which originated from the same agro-ecological region (Sakarya). The most similar F. culmorum isolates were F20 and
\end{abstract}


F21 (81.1\%), which were from different geographic regions (Bilecik and Uşak, respectively). Moreover, interspecific variation between the two species was determined to be 86.3 to $93.3 \%$. Cluster analysis generated two branched groups, each containing isolates of one species, except F13 of $F$. culmorum. The sequencing of stable and reproducible monomorphic and polymorphic RAPD markers indicated that the Fusarium genome shared high similarity (105-625 bit scores) with the genomes of other organisms as well as with the $F$. graminearum reference genome.

Key words: Fusarium head blight; F. graminearum; F. culmorum; Genotyping; Random-amplified polymorphic DNA

\section{INTRODUCTION}

Wheat is one of the most important cereal crops worldwide. In a 2009 ranking of cereal crops in Turkey, wheat was first in terms of production, area harvested, and seed production (FAO, 2012). Wild-type cultivated cereals, especially wheat and barley, are still distributed along the Fertile Crescent, which includes part of south eastern Turkey, where agriculture was first initiated (Harlan and Zohary, 1966). However, cultivated cereals are susceptible to many diseases. Among them Fusarium head blight (FHB) is one of the most devastating diseases, and particularly affects wheat. Many Fusarium spp cause the disease in Turkey and other countries worldwide (Champail et al., 2004). Fusarium graminearum and F. culmorum are the most common causal agents of FHB (Miedaner et al., 2001). FHB epidemics cause both severe yield losses and reduction in quality. Mycotoxins produced by the pathogens effectively spread the disease. Moreover, they have long-term harmful effects on both humans and animals entering the food chain. In addition to their carcinogenic effects, they inhibit ribosomal protein synthesis (Gutleb et al., 2002). Research has recently focused on the molecular characterization of causal agents and the production of mycotoxins to improve pathogen recognition (Saharan et al., 2007; Haratian et al., 2008; Pandolfi et al., 2010).

Typing or genotyping, referred to as molecular epidemiology, is as important as the study of global epidemiology of pathogens. Unless the genetic variants collected from various regions of the world can be identified, the long-term struggle with plant pathogens will continue. Nevertheless, our knowledge about the genetic diversity of plant pathogens is limited (Virdi and Sachdeva, 2005). Therefore, understanding the genetic variation of Fusarium spp associated with FHB will improve disease-control strategies. Owing to a lack of specific and effective fungicides, the development and selection of resistant cultivars have become an important strategy in disease control and cereal crop breeding programmes (Mesterházy, 1995; Saharan et al., 2004). The detection of Fusarium spp among field pathogens, their identification, and the determination of the genetic diversity of their isolates are methodological approaches. The identification of Fusarium spp based on morphological characters using traditional techniques is influenced by host age, inoculum quality, and environmental conditions (Champail et al., 2004; Saharan et al., 2004). In addition, traditional techniques are time-consuming and laborious. Therefore, molecular techniques have recently become a promising and versatile tool, especially for the detection of genetic variation (Demir et al., 2010; Abadio et al., 2012; Memati et al., 2012). Because these techniques are reliable, fast, sensitive, and suitable, they can replace traditional methods. Some of them - for example, random-amplified polymorphic DNA (RAPD), microsatellite polymerase chain reaction (PCR), 
and inter-simple sequence repeats - are used to determine intra- and interspecific genetic variations in Fusarium spp (Carter et al., 2002; Mishra et al., 2003; Heng et al., 2012).

Although FHB can be diagnosed on all small-grain crops, it has been reported most frequently on wheat in Turkey (Mamluk et al., 1997). Tunali et al. (2006a,b) obtained 116 singlespore isolates belonging to Fusarium - including F. graminearum and F. culmorum - from wheat spikes in the north central parts of Anatolia and Marmara region between 2000 and 2005. Three of these species (F. culmorum, F. graminearum, and F. pseudograminearum) were observed as the most aggressive pathogens, and they reduced vitality under greenhouse conditions. The authors also reported that $F$. graminearum was the primary pathogen responsible for FHB and that the disease incidence was positively correlated with the content of deoxynivalenol that accumulated during that period of time. Gürel et al. (2010) determined the genetic variation of 15 Turkish $F$. culmorum isolates from nine agro-ecological regions using repetitive DNA-based fingerprinting methods. This report was the first to describe the molecular characterization of these isolates and their relationship with the wide geographic distribution of that species. Arici and Koç (2010) also investigated the genetic variation of 10 isolates belonging to $F$. graminearum and $F$. culmorum from the Adana region using RAPD markers. Moreover, chemotyping of $11 \mathrm{~F}$. graminearum and $21 \mathrm{~F}$. culmorum isolates from various agro-ecological regions of Turkey has been carried out using PCR analysis (Yörük and Albayrak, 2012).

The identification of Fusarium spp and their produced mycotoxins are important in treatment strategies for FHB and the development of new species-specific diagnostics in Turkey. In this study, we aimed to 1) analyze the genetic variation of F. graminearum and F. culmorum isolates collected from various regions of Turkey, 2) detect nucleotide sequences that are sequence characterized amplified regions (SCAR) markers in the genomes of these pathogens, and 3) investigate the homologies of these sequences with the genomes of other organisms as well as the reference genome of $F$. graminearum.

\section{MATERIAL AND METHODS}

\section{Fungal material and genomic DNA extraction}

Seventeen isolates of Fusarium, including 5 F. graminearum and 12 F. culmorum produced from single spores (Bentley et al., 2006) were provided by Dr. Berna Tunali (Department of Plant Protection, Agricultural Faculty, Samsun Ondokuz Mayis University; Table 1). All isolates were grown on potato dextrose agar plates at $25^{\circ} \mathrm{C}$ for 6 days. Genomic DNA was extracted using a genomic DNA isolation kit (Macherey-Nagel, A026218, Germany) based on the established cetyltrimethylammonium bromide procedure.

\section{Identification of trichothecene-producing groups and species}

Primer set Tox5-1/Tox5-2 (5'-GCTGCTCATCACTTTGCTCAG/CTGATCTGGTCACG CTCATC-3') was used to amplify the trichodiene synthetase (tri5) gene (Table 2) to identify trichothecene-producing Fusarium spp (Hue et al., 1999). PCR was carried out in a volume of $25 \mu \mathrm{L}$ containing $50 \mathrm{ng}$ genomic DNA, $1.5 \mathrm{mM} \mathrm{MgCl}$, 1 X PCR buffer, 10 pmol of each primer, $0.1 \mathrm{mM}$ of each deoxyribonucleoside triphosphate, and 1 U Taq DNA polymerase (9PIM829; Promega, USA). PCR amplification was performed by predenaturation at $94^{\circ} \mathrm{C}$ for $5 \mathrm{~min}$, followed by 35 
Table 1. Hosts and geographic origins of Fusarium culmorum and F. graminearum isolates used in this study.

\begin{tabular}{|c|c|c|c|}
\hline Isolate & Species & Host & Geographic origin \\
\hline F2 & F. culmorum & Wheat & Sakarya \\
\hline F3 & F. culmorum & Wheat & Konya Merkez \\
\hline F4 & F. culmorum & Wheat & Sakarya \\
\hline F5 & F. graminearum & Wheat & Sakarya \\
\hline F6 & F. graminearum & Wheat & Sakarya \\
\hline F7 & F. graminearum & Wheat & Sakarya \\
\hline F8 & F. graminearum & Wheat & Sakarya \\
\hline F9 & F. graminearum & Wheat & Balıkesir \\
\hline F10 & F. culmorum & Wheat & Bilecik \\
\hline F13 & F. culmorum & Wheat & Osmaneli/Bilecik \\
\hline F14 & F. culmorum & Wheat & Bilecik \\
\hline F15 & F. culmorum & Wheat & Boyabat/Sinop \\
\hline F17 & F. culmorum & Wheat & Konya Merkez \\
\hline F19 & F. culmorum & Wheat & Sarayönü/Konya \\
\hline F20 & F. culmorum & Wheat & Bilecik \\
\hline F21 & F. culmorum & Wheat & Uşak \\
\hline F24 & F. culmorum & Wheat & Cumra/Konya \\
\hline
\end{tabular}

cycles of amplification at $94^{\circ} \mathrm{C}$ for $1 \mathrm{~min}, 68^{\circ} \mathrm{C}$ for $1 \mathrm{~min}, 72^{\circ} \mathrm{C}$ for $1.5 \mathrm{~min}$, and a final extension at $72^{\circ} \mathrm{C}$ for $10 \mathrm{~min}$. F. graminearum isolates were confirmed via Fg16F/Fg16R (5'-CTCCGGATA TGTTGCGTCAA-3'/5'-GGTAGGTATCCGACATGGCAA-3') and UBC85F/UBC85R (5'-GCA GGGTTTGAATCCGAGAC-3'/5'-AGAATGGAGCTACCAACGGC-3') primer sets designed by Nicholson et al. (1998) and Schilling et al. (1996), respectively. Similarly, F. culmorum isolates were verified via Fc01F/Fc01R (5'-ATGGTGAACTCGTCGTGGC-3'/5'-CCCTTCTTACGCCA ATCTCG-3') and OPT18F/OPT18R (5'-GATGCCAGACCAAGACGAAG-3'/5'-GATGCCAGA CGCACTAAGAT-3') primers, also designed by Nicholson et al. (1998) and Schilling et al. (1996), respectively. PCR mixing and thermal cycling were carried out as previously described, with the exception of the annealing temperature, which was adjusted to $61^{\circ} \mathrm{C}$. Amplification products were separated with electrophoresis on $1.5 \%$ agarose gel and visualized under ultraviolet light after staining with ethidium bromide.

\section{RAPD analysis}

In this study, 65 random-10-mer oligonucleotide primers from Operon Technologies (USA) were examined through RAPD analysis (see Table 2). Amplification reactions were carried out in volumes of $25-\mu \mathrm{L}$ containing $50 \mathrm{ng}$ genomic DNA, 1X PCR buffer, $2.5 \mathrm{mM}$ $\mathrm{MgCl}_{2}, 0.4 \mathrm{mM}$ of each deoxyribonucleoside triphosphate, $10 \mathrm{pmol}$ primer and $1 \mathrm{U}$ Taq DNA polymerase (Promega). The thermal cycling conditions for all primers consisted of DNA denaturation at $94^{\circ} \mathrm{C}$ for $5 \mathrm{~min} ; 4$ cycles of $1.5 \mathrm{~min}$ at $94^{\circ} \mathrm{C}, 1.5 \mathrm{~min}$ at $37^{\circ} \mathrm{C}$, and $3 \mathrm{~min}$ at $72^{\circ} \mathrm{C}$; followed by 40 cycles of $1 \mathrm{~min}$ at $94^{\circ} \mathrm{C}, 1 \mathrm{~min}$ at $37^{\circ} \mathrm{C}, 2 \mathrm{~min}$ at $72^{\circ} \mathrm{C}$, and a final extension at $72^{\circ} \mathrm{C}$ for $10 \mathrm{~min}$. RAPD products were electrophoresed on $1.7 \%$ agarose gel. RAPD assays for each of the primers were carried out three times to verify reproducible amplicons.

\section{Statistical analysis}

RAPD markers were visually scored as the presence (1) or absence (0) of a band. A similarity matrix was constructed from these data using Nei-Li's coefficient (Nei and Li, 1979). A dendrogram was generated according to the cluster analysis of RAPD markers using the unweighted pair group method with arithmetic average algorithm (UPGAM) in MVSP 3.1. 


\begin{tabular}{|c|c|c|c|c|c|}
\hline Primer & Sequence (5'-3') & Primer & Sequence (5'-3') & Primer & Sequence (5'-3') \\
\hline OPA02 & TGCCGAGCTG & OPC19 & GTTGCCAGCC & OPF06 & GGGAATTCGG \\
\hline OPA06 & GGTCCCTGAC & OPD01 & ACCGCGAAGG & $\mathrm{OPG} 02$ & GGCACTGAGG \\
\hline OPA09 & GGGTAACGCC & OPD02 & GGACCCAACC & OPG03 & GAGCCCTCCA \\
\hline OPA13 & CAGCACCCAC & OPD03 & GTCGCCGTCA & OPG07 & GAACCTGCGG \\
\hline OPA 18 & AGGTGACCGT & OPD04 & TCTGGTGAGG & OPG10 & AGGGCCGTCT \\
\hline OPB03 & CATCCCCCTG & OPD07 & TTGGCACGGG & OPG11 & TGCCCGTCGT \\
\hline OPB05 & TGCGCCCTTC & OPD08 & GTGTGCCCCA & OPG13 & CTCTCCGCCA \\
\hline OPB06 & TGCTCTGCCC & OPD13 & GGGGTGACGA & OPG16 & AGCGTCCTCC \\
\hline OPB07 & GGTGACGCAG & OPD14 & CTTCCCCAAG & OPG17 & ACGACCGACA \\
\hline OPB09 & TGGGGGACTC & OPD17 & TTTCCCACGG & OPZ02 & CCTACGGGGA \\
\hline OPB10 & CTGCTGGGAC & OPD20 & ACCCGGTCAC & OPZ03 & CAGCACCGCA \\
\hline OPB13 & TTCCCCCGCT & OPE01 & CCCAAGGTCC & OPZ04 & AGGCTGTGCT \\
\hline OPB14 & TCCGCTCTGG & OPE02 & GGTGCGGGAA & OPZ07 & CCAGGAGGAC \\
\hline OPB19 & ACCCCCGAAG & OPE03 & CCAGATGCAC & OPZ08 & GGGTGGGTAA \\
\hline OPC02 & GTGAGGCGTC & OPE06 & AAGACCCCTC & OPZ09 & CACCCCAGTC \\
\hline OPC04 & CCGCATCTAC & OPE13 & CCCGATTCGG & OPZ10 & CCGACAAACC \\
\hline OPC05 & GATGACCGCC & OPE14 & TGCGGCTGAG & OPZ12 & TCAACGGGAC \\
\hline OPC07 & GTCCCGACGA & OPF01 & ACGGATCCTG & OPZ15 & CAGGGCTTTC \\
\hline OPC08 & TGGACCGGTG & OPF02 & GAGGATCCCT & OPZ16 & TCCCCATCAC \\
\hline OPC09 & CTCACCGTCC & OPF03 & CCTGATCACC & OPZ19 & GTGCGAGCAA \\
\hline OPC14 & TGCGTGCTTG & OPF04 & GGTGATCAGG & OPZ20 & ACTTTGGCGG \\
\hline OPC17 & TTCCCCCCAG & OPF05 & CCGAATTCCC & & \\
\hline
\end{tabular}

\section{Screening of stable and reproducible polymorphic and monomorphic RAPD markers}

Distinctive stable and reproducible polymorphic and monomorphic RAPD markers with the potential to differentiate the two species were detected. These markers were then purified from agarose gels using a High Pure PCR Product Purification Kit (Roche, 11732668001, Switzerland). The amplicons were cloned into plasmids using a p-GEM Easy Vector System (Promega, TM042) and then sequenced with universal M13 primers. The sequencing process was carried out based on a chain termination method. Nucleotide sequences were then monitorized as chromatograms using ChromasPro and subjected to homology analysis. Sequence similarity (e value $<0.05$; bit score $>50$ ) was searched with nucleotide Basic Local Alignment Search Tool (BLASTN) through the National Center for Biotechnology Information (NCBI) network (Altschul et al., 1997). Some criteria in particular, such as nucleotide collection and highly similar sequences, were considered within BLASTN.

\section{RESULTS}

\section{Identification of trichothecene-producing groups and species}

In PCR analysis, the Tox5-1/Tox5-2 primer produced a 658-bp fragment in all isolates (Figure 1A). Therefore, we determined that 17 isolates had trichothecene-producing potentials.

$\mathrm{Fg} 16 \mathrm{~F} / \mathrm{Fg} 16 \mathrm{R}$ generated a common band of $450 \mathrm{bp}$ (Figure 1B), whereas UBC85F/ UBC85R amplified a band of $332 \mathrm{bp}$ (data not shown) in only 5 isolates (F5-F9). Thus, we confirmed 5 isolates as F. graminearum. Similarly, the Fc01F/Fc01R and OPT18F/OPT18R primer pairs yielded product sizes of $570 \mathrm{bp}$ (Figure 1B) and $472 \mathrm{bp}$ (data not shown), respectively. These PCR results indicated that 11 isolates (not including F13) belonged to F. culmorum. Thus, we identified all isolates at the species and group levels. 


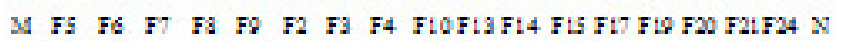

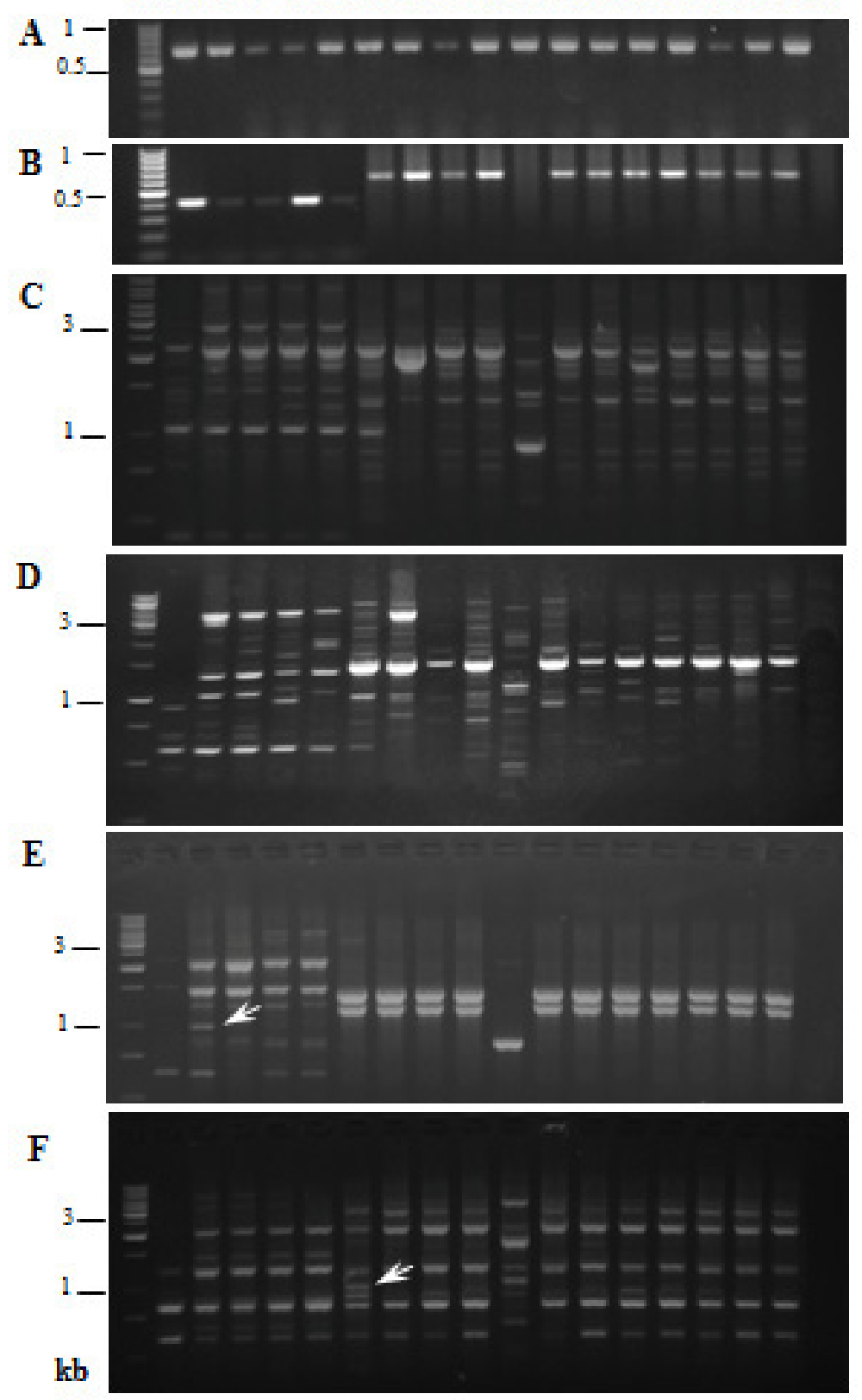

Figure 1. DNA amplification products (A) of a 658-bp fragment with Tox5-1/Tox5-2 primer in all isolates, (B) of 450 bp with Fg16F/Fg16R primer specific to Fusarium graminearum isolates (F5, F6, F7, F8, F9) and of 570 bp with Fc01F/ Fc01R primer specific to F. culmorum isolates (F2, F3, F4, F10, F14, F15, F17, F19, F20, F21, and F24). RAPD profiles of 5 F. graminearum and 12 F. culmorum (F2, F3, F4, F10, F13, F14, F15, F17, F19, F20, F21, and F24) isolates amplified with (C) OPC07, (D) OPC17, (E) OPG11, and (F) OPG13 primers. White arrows indicate polymorphic markers. Lane $M$ $=100-\mathrm{bp}(\mathbf{A}$ and $\mathbf{B})$ and 1-kb ladder $(\mathbf{C}, \mathbf{D}, \mathbf{E}, \mathbf{F})$ molecular weight standards; lane $N=$ negative control. 


\section{RAPD analysis}

Sixty-five 10-mer oligonucleotide primers were examined for amplification of RAPD markers of 16 isolates. Genomic DNA of isolate F13 displayed different amplification patterns with all primers. Moreover, neither Fc01F/Fc01R nor OPT18F/OPT18R generated any DNA fragments in species-specific PCR of this isolate. Hence, F13 was excluded from the evaluation of RAPD findings and generation of relationships. Fifty primers produced strong and reproducible DNA amplicons. The highest number of amplification patterns was observed using OPC17 (Figure 1C). The remaining primers generated either similar amplicons or no amplification products. In total, 1200 fragments were scored for multivariate analysis, 311 of which were determined to be polymorphic and unique to the isolates (Figure 1D-F). The RAPD amplicon sizes ranged from 0.3 to $4 \mathrm{~kb}$ among 5 F. graminearum isolates and from 0.2 to $5 \mathrm{~kb}$ among 11 F. culmorum isolates. According to Nei-Li's coefficient, the mean value of genetic similarity was $61.5 \%$ for $F$. graminearum isolates and $65 \%$ for F. culmorum isolates (Table 3 ). The similarity coefficient among $F$. graminearum and $F$. culmorum isolates ranged from 43 to $76.1 \%$ and 49 to $81.1 \%$, respectively. Isolates F6 and F7 (F. graminearum) were the most genetically related isolates and originated in the same geographic region (Sakarya). Similarly, F20 and F21 (F. culmorum isolates), which belonged to geographically different regions (Bilecik and Uşak, respectively) were determined to be highly similar (81.1\%). Moreover, interspecific variation between two species ranged from 86.3 to $93.3 \%$. Although F7 (F. graminearum) and F2 (F. culmorum) were genetically the most similar $(13.7 \%)$ isolates belonging to the same geographic region (Sakarya), F5 (F. graminearum) and F19 (F. culmorum) were dissimilar (6.7\% similarity) and originated from different regions (Sakarya and Sarayönü/Konya, respectively). F5 and F13 were the most distant isolates, and F13 was excluded from data analysis.

Table 3. Similarity matrix belong to 16 isolates of Fusarium culmorum and F. graminearum.

\begin{tabular}{|c|c|c|c|c|c|c|c|c|c|c|c|c|c|c|c|c|}
\hline & F5 & F6 & F7 & F8 & F9 & $\mathrm{F} 2$ & F3 & F4 & F10 & F14 & F15 & F17 & F19 & F20 & F21 & $\mathrm{F} 24$ \\
\hline F5 & 1.000 & & & & & & & & & & & & & & & \\
\hline F6 & 0.446 & 1.000 & & & & & & & & & & & & & & \\
\hline F7 & 0.444 & 0.761 & 1.000 & & & & & & & & & & & & & \\
\hline F8 & 0.430 & 0.673 & 0.691 & 1.000 & & & & & & & & & & & & \\
\hline F9 & 0.437 & 0.665 & 0.727 & 0.750 & 1.000 & & & & & & & & & & & \\
\hline F2 & 0.096 & 0.132 & 0.137 & 0.127 & 0.135 & 1.000 & & & & & & & & & & \\
\hline F3 & 0.090 & 0.117 & 0.134 & 0.115 & 0.129 & 0.684 & 1.000 & & & & & & & & & \\
\hline F4 & 0.075 & 0.118 & 0.117 & 0.129 & 0.127 & 0.708 & 0.683 & 1.000 & & & & & & & & \\
\hline F10 & 0.082 & 0.122 & 0.121 & 0.120 & 0.125 & 0.724 & 0.721 & 0.784 & 1.000 & & & & & & & \\
\hline F14 & 0.075 & 0.118 & 0.111 & 0.107 & 0.108 & 0.680 & 0.709 & 0.732 & 0.794 & 1.000 & & & & & & \\
\hline F15 & 0.074 & 0.120 & 0.118 & 0.115 & 0.122 & 0.613 & 0.622 & 0.691 & 0.725 & 0.734 & 1.000 & & & & & \\
\hline F17 & 0.071 & 0.080 & 0.095 & 0.087 & 0.107 & 0.491 & 0.545 & 0.545 & 0.569 & 0.589 & 0.607 & 1.000 & & & & \\
\hline F19 & 0.067 & 0.103 & 0.124 & 0.111 & 0.112 & 0.602 & 0.633 & 0.649 & 0.690 & 0.710 & 0.705 & 0.602 & 1.000 & & & \\
\hline F20 & 0.070 & 0.109 & 0.104 & 0.110 & 0.114 & 0.630 & 0.622 & 0.697 & 0.704 & 0.733 & 0.808 & 0.610 & 0.741 & 1.000 & & \\
\hline F21 & 0.071 & 0.116 & 0.108 & 0.114 & 0.115 & 0.613 & 0.622 & 0.675 & 0.685 & 0.724 & 0.794 & 0.590 & 0.690 & 0.811 & 1.000 & \\
\hline F24 & 0.088 & 0.126 & 0.131 & 0.124 & 0.132 & 0.598 & 0.640 & 0.659 & 0.682 & 0.719 & 0.688 & 0.611 & 0.717 & 0.742 & 0.739 & 1.000 \\
\hline
\end{tabular}

Cluster analysis generated two groups, each containing isolates of one species with the exception of F13, which was of F. culmorum (Figure 2). As shown in the dendrogram, group I was larger cluster than group II because it consisted of 11 isolates, whereas group II contained only 5 isolates. Moreover, both groups were subdivided further into two subgroups. Isolates F5 (F. graminearum) and F17 (F. culmorum) were absent from these subgroups. They were related to their main groups. 


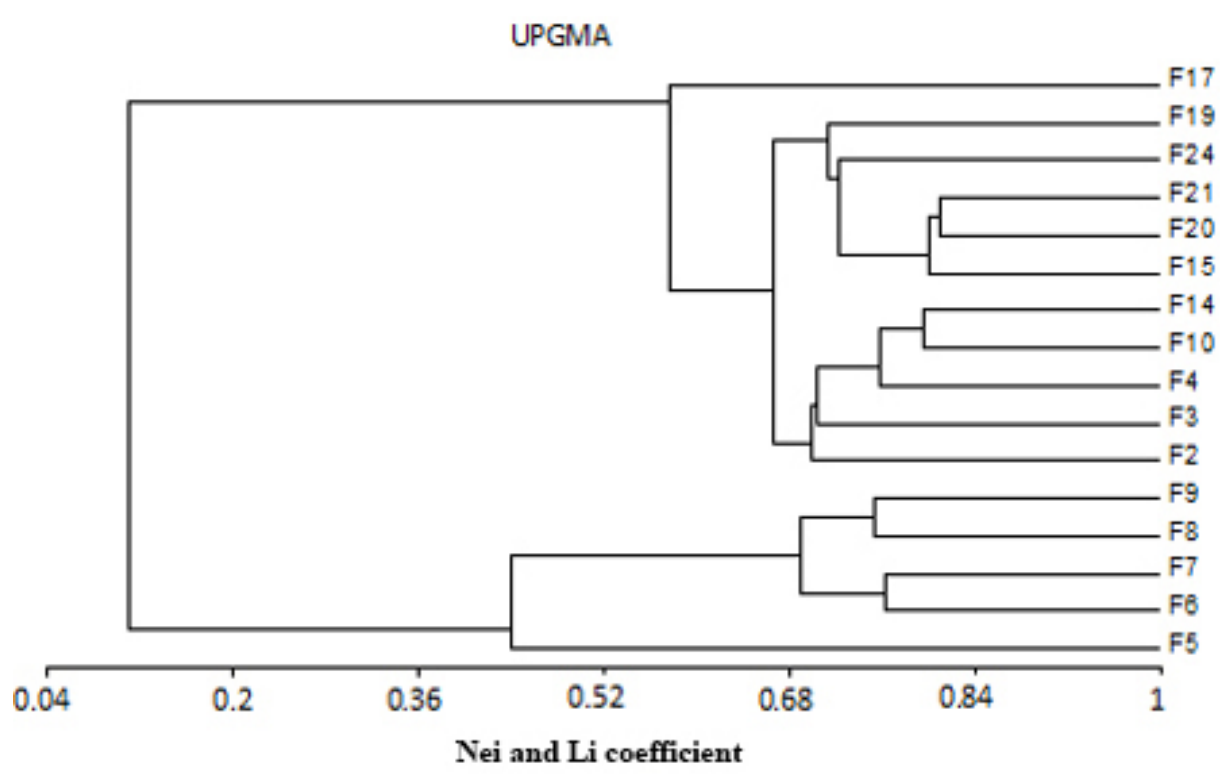

Figure 2. Cluster analysis of genetic similarity obtained from 16 isolates of Fusarium culmorum and F. graminearum.

\section{Screening of stable and reproducible polymorphic and monomorphic RAPD markers}

Owing to their reproducibility and stability, 22 polymorphic and 4 monomorphic RAPD markers were selected as SCAR markers to generate detailed sequence information. After screening the sequence data of markers using ChromasPro, we evaluated high-quality signal peaks in chromatograms. Seven of the polymorphic and all monomorphic RAPD markers showed significant nucleotide sequence homologies to the genomes of various organisms as well as to the F. graminearum genome registered in NCBI (Table 4). All similarity indexes had acceptable e values $(<0.05)$ and bit scores $(>50)$. Among polymorphic RAPD markers, the insert from 2.17.D07 and 7.08.A09 clones had identical nucleotide sequences with a polymerase protein and coat protein in hepatitis B virus. In addition, the insert from the 6.13.D08 clone shared identical nucleotide sequences with the marenostrin protein in Homo sapiens. Another insert from 22.06.E14 showed 20\% nucleotide similarity with a transfer RNA (tRNA)-Lys and maturase K (matK) in Nymphaea macrosperma. Moreover, the BLASTN analysis indicated that three inserts from 20.24.D02, 14.04.C17, and 16.17.C17 clone sequences were similar $(53,81$, and $91 \%$, respectively) to $\beta$-lactamase TEM-116 and blaCTX-M-15 gene sequences. In addition to polymorphic markers, all monomorphic RAPD markers had significant sequence homologies with the genomes of other organisms (see Table 4). The insert from the D3G clone shared sequence similarity (25\%) with PH-1 hypothetical mRNA in Gibberella zeae (asexual F. graminearum). The D3C clone insert displayed nucleotide sequence similarity (17\%) with a predicted protein-coding region in Nectria haematococca (asexual name F. solani). Finally, the inserts from the G11.1C and G11.2C clones revealed 10 and $15 \%$ nucleotide homologies with genomic fragments of Lepilemur dorsalis and 18S ribosomal DNA (rDNA) of uncultured Trebouxia photobiont, respectively. 


\begin{tabular}{|c|c|c|c|c|}
\hline Marker & Associated organism & Accession No. & Product & e value \\
\hline 2.17.D07 & Hepatitis B virus & FJ621799.1 & Polymerase $(\mathrm{P})$ protein & 0.0 \\
\hline 7.08.A09 & Hepatitis B virus & AB263407.1 & Coat protein & 0.0 \\
\hline 6.13.D08 & Homo sapiens & NG_007871 & Marenostrin protein & 0.0 \\
\hline 22.06.E14 & Nymphaea macrosperma & DQ1 185540.1 & tRNA-Lys and maturase $\mathrm{K}$ & $5 e-46$ \\
\hline 20.24.D02 & Aeromonas jandaei & FJ767909.1 & $\beta$-lactamase TEM-116 & $2 \mathrm{e}-94$ \\
\hline 14.04.C17 & Klebsiella pneumoniae & GQ845086 & $\beta$-lactamase blaCTX-M-15 & $2 \mathrm{e}-177$ \\
\hline 16.17.C17 & Enterobacter $\mathrm{sp}$ & EU531513.1 & $\beta$-lactamase blaCTX-M-28 & $5 e-111$ \\
\hline $\mathrm{D} 3 \mathrm{G}$ & Gibberella zeae & XM 389641.1 & Hypothetical protein & $5 e-61$ \\
\hline $\mathrm{D} 3 \mathrm{C}$ & Nectria haematococca & XM_003046704.1 & Predicted protein & $4 e-37$ \\
\hline $\mathrm{G} 11.1 \mathrm{C}$ & Lepilemur dorsalis & $\mathrm{AJ} 2 \overline{4} 4005.1$ & Unknown & $1 e-33$ \\
\hline $\mathrm{G} 11.2 \mathrm{C}$ & Uncultured Trebouxia photobiont & AJ969582 & $18 \mathrm{~S}$ ribosomal RNA & $1 e-36$ \\
\hline
\end{tabular}

\section{DISCUSSION}

The occurrence of a disease is closely related not only to plant-fungal pathogen interactions but also to environmental conditions (Gutleb et al., 2002). More recent global climatic changes can contribute to the spread of a disease from geographical area in which it was not previously observed to regions throughout the world (Saharan et al., 2004). Therefore, strategies for combating disease must be revised. Controlling the disease and the genotyping of causal agents depend on accurate diagnosis (Ward et al., 2004). Conventional characterization methods for Fusarium spp are time-consuming, inconclusive, and laborious. Conversely, nucleic acid-based molecular techniques are reliable, fast, and suitable for diagnosis, identification of pathogens or their specific markers, and genotyping. In this study, 5 F. graminearum and $11 \mathrm{~F}$. culmorum isolates identified using conventional methods were confirmed through PCR analysis of SCAR markers. SCAR analysis is a powerful tool for definitive diagnosis at the species level. F. culmorum F13 was excluded from the comparison of all isolates because its genomic DNA was not amplified using species-specific primers.

These findings can be explained in that F13 contains genomic alterations such as transition/transversion or rearrangements such as insertion/deletion in the primer annealing sites. Carter et al. (2000) excluded WL6 and MK4 isolates from their Fusarium analysis according to SCAR results. They have shown that both of the isolates are classified as F. napiforme and F. fujikuroi using internal transcribed spacer sequence analysis. These outcomes cause uncertainty about the reliability of conventional methods for the diagnosis or identification of pathogenic fungi. As has already been stated in the literature, findings obtained with conventional methods must be confirmed using molecular techniques. Therefore, combining conventional techniques with molecular methods provides accurate diagnosis of the disease and proper identification of fungal organisms.

The RAPD technique has been effective for investigating genetic diversity in Fusarium spp. Walker et al. (2001) and Miedaner et al. (2001) reported high levels of genetic variation between F. culmorum and F. graminearum isolates. On the contrary, various studies (Quellet and Seifert, 1993; Carter et al., 2000; Saharan et al., 2007) have indicated a relatively low amount of genetic diversity among $F$. graminearum strains. In this study, intra- and interspecific genetic variations of Turkish $F$. graminearum and $F$. culmorum isolates were investigated using an RAPD method. Interspecific similarity among the isolates ranged from 6.7 to $13.7 \%$ according to the similarity matrix. Moderate and low levels of intraspecific 
variation were detected among F. culmorum (18.9 to 51\%) and F. graminearum (23.9 to 57\%) isolates. Cluster analysis of RAPD data showed that the isolates were divided into two main clusters (groups I and II), each containing isolates of one species, with the exception of F13 F. culmorum. Although four of $F$. graminearum isolates were collected from the same region (Sakarya), they showed moderate variation (23.9 to 57\%). With the exception of F5, they were separated into two subgroups. Whereas F6 and F7 isolates from Sakarya were divided into one subgroup, F8 joined F9 from Balikesir in the other. Although F5 was obtained from Sakarya, it was excluded from both subgroups. Genetically, the most similar F. graminearum isolates were F6 and F7, and they originated from the same geographic region.

According to Nei-Li's coefficient, similarity among F. culmorum isolates was 13.7 to $81.1 \%$, including F13. Isolates F20 and F21 were the most similar, and they originated from Bilecik and Uşak, respectively. The dendrogram indicated that all Marmara region isolates except F20 clustered in the same subgroup. The other subgroup showed heterogeneity in terms of geographic origin. This subgroup also contained the isolates with the highest similarity value - for example, 81.1\% (between F20 and F21) and 80.9\% (between F15 and F20). The RAPD dendrogram illustrated that $F$. culmorum isolates, except F17, were divided into two subgroups. $F$. graminearum isolates had a higher polymorphism potential than those of $F$. culmorum. We detected no correlation between genotypes and geographic origin that was influenced by $F$. graminearum because the sampling number was very low. The number of $F$. culmorum isolates was sufficient to evaluate this correlation, and we found that isolates present in group I showed geographic heterogeneity.

Many studies have reported that some isolates belonging to the same geographic region are found in different clusters, and others from different regions are grouped in the same cluster (Carter et al., 2002; Saharan et al., 2007). Determining the correlation between polymorphism/similarity and geographic origin of Fusarium isolates is extremely difficult. Considerably high intraspecific variation (60 to 100\%) exists among the isolates of worldwide collections even within populations from individual fields (Miedaner et al., 2008). However, compared to this high diversity, a moderate level of interspecific variation (18.9 to 57\%) was presented among Turkish Fusarium isolates obtained from nine regions. These findings suggest that the genetic composition of the Turkish Fusarim population has stability. Saharan et al. (2007) concluded that genetic diversity is also associated with variation in climatic conditions, farm practices, and crop rotations. Moreover, sexual or parasexual recombination is responsible for intraspecific variation within F. graminearum isolates, whereas asexual or parasexual recombination accounts for variability within F. culmorum isolates (Miedaner et al., 2001). Gürel et al. (2010) reported relatively high genetic variability (11.3 to 84.2\%) within $F$. culmorum isolates from Turkey by using repetitive DNA-based fingerprinting methods. Some F. culmorum isolates examined in the present study were also used by Gürel et al. (2010). A comparison of repetitive element palindromic and RAPD dendrograms has shown that F2, F3, F4, F10, and F14 cluster in the same subgroup, whereas F15, F19, F20, and F21 cluster in the other subgroup. These compatible data indicate that selected molecular marker techniques are reliable for genotyping.

Genotyping of closely related Fusarium isolates can contribute to diagnosis, disease control strategies, and selection of suitable plant breeding programmes related to FHB. The development of SCAR markers derived from RAPD is an important advancement for species-specific diagnosis. SCAR markers have been used to identify pathogenic fungi and dis- 
tinguish wild-type and mutant organisms. These markers have also provided knowledge about nucleotide sequence homology and orthologous genes among genomes (Nicholson et al., 1998; Adinolfi et al., 2007; Saharan et al., 2007). They are frequently used in the molecular characterization of Fusarium at the genus and species levels (Schilling et al., 1996; Haratian et al., 2008).

Genetic homology is evaluated on the basis of the detection of both amino acid and nucleic acid sequence similarity by using whole-genome scanning methods. The fact that two organisms contain homologous or highly similar sequences indicates that they originated from a common ancestor. In particular, the determination of sequence homology provides data not only for the construction of evolutionary trees but also for the development of new model organisms that can be used in basic research in the future. In this study, we examined the utility of RAPD marker sequences as SCAR markers. We also searched for similarities between these sequences and those of the genomes of other organisms and the $F$. graminearum genome. In this study, stable, reproducible, polymorphic RAPD markers amplified in F. graminearum or F. culmorum genomes showed significant nucleotide sequence similarities or homologies with the genomes of other organisms, especially of unrelated species. The high level of homology demonstrated that these sequences were evolutionary conserved from viruses to humans. In particular, the similarity between RAPD markers obtained from F. culmorum and antibiotic resistant gene sequences in bacteria is crucial with regard to natural selection.

The most remarkable finding in the BLASTN analysis was the high nucleotide sequence similarity between marker 6.13.D08 of isolate F13 and the Mediterranean fever gene on chromosome $16 \mathrm{H}$. sapiens. The marenostrin protein is the final product of this gene, and it is related to familial Mediterranean fever. Although we excluded isolate F13 from diversity analysis, we found that polymorphic marker $6.13 . \mathrm{D} 08$ had 93\% coverage and 98\% maximum identity values to this gene. For this reason, F13 can be used as model organism in molecular studies related to Mediterranean fever. Another important similarity (coverage: $91 \%$, maximum identity: 90\%) was detected between the 16.17.C17 marker amplified in F17 and $\beta$-lactamase bla CTX-M-28 type of Enterobacter spp. In addition, the $\beta$-lactamase bla CTX-M-15 type of Klebsiella pneumoniae shared homology with the 14.04.C17 marker in F4 of the same fungal pathogen. Both the 2.17.D07 and 7.08.A09 markers of F. culmorum and F. graminearum showed high similarity (coverage: $81 \%$, maximum identity: $95 \%$; coverage: $75 \%$, maximum identity: $97 \%$, respectively) to the polymerase protein and coat protein of hepatitis B virus, respectively. The similarity of the 22.06.E14 marker sequence of $F$. graminearum to the tRNALys and matK gene sequences of $N$. macrosperma was also a remarkable finding in terms of calculated coverage and maximum identity values ( 81 and $95 \%$, respectively).

In addition to these BLASTN results, similarities between stable, strong monomorphic RAPD marker sequences, which separated two Fusarium spp, and genomes of other organisms were observed. Despite their relatively low coverage value, these marker sequences shared significant maximum identity, even identical nucleotide sequences, with part of genome sequences of other organisms. Homology values of monomorphic marker sequences were lower than those of polymorphic ones. The monomorphic D3G marker amplified in $F$. graminearum resembled the PH-1 hypothetical partial mRNA of G. zeae (anamorph $F$. graminearum) registered in the database (NCBI, 2012). Despite having a low coverage value (25\%), the maximum identity was $100 \%$. This result indicates that the generated D3G marker contains common sequences with $F$. graminearum genes published in the NCBI database. The D3C marker of F. culmorum was similar (coverage: $17 \%$, maximum identity: $87 \%$ ) to the mpVI 77 - 
13-4 predicted protein coding genomic region of $N$. haematococca (asexual name of $F$. solani). This finding is also valuable in terms of determining conserved sequences among Fusarium spp. Moreover, a short genomic region associated with unrelated species was detected in this study. For example, G11.2C marker sequences showed similarity with the available uncultured T. photobiont $18 \mathrm{~S}$ rDNA region (coverage: $15 \%$; maximum identity: $100 \%$ ). The $F$. culmorum genome had sequences closely related to those within algal genomes. Knowledge from both polymorphic and monomorphic RAPD markers produced within isolates belonging to two Fusarium spp may be useful for evaluating evolutionary relationships.

In the present study, we identified 17 isolates of Fusarium, including 5 F. graminearum and 12 of F. culmorum at the species level by using PCR-based techniques. We realized that the number of $F$. graminearum isolates was very low; we decided that identifying them was important. We reliably confirmed all isolates in a short time at the species level and determined the relationship among them using RAPD analysis. We found a moderate level of intraspecific genetic variation. Simultaneously, we investigated the potential of the produced RAPD markers to be used as SCAR markers. However, 11 RAPD markers were not allele specific. Hence, they cannot be used as species-specific SCAR markers. Nevertheless, marker sequence information is useful for molecular biologists. Therefore, the sequence of the D3G RAPD marker, generated from the $F$. graminearum $\mathrm{F} 7$ isolate was registered in the NCBI database (GenBank) under accession No. JN638063. The remaining sequence data will also be deposited in the database. We believe that the findings of this research will contribute to genome studies of Fusarium spp, especially F. culmorum.

\section{ACKNOWLEDGMENTS}

Research supported by the Research Fund of the Istanbul University (\#T1494/04092007 and \#5765). The authors thank Dr. Berna Tunali from the Department of Plant Protection, Agricultural Faculty, Samsun Ondokuz Mayis University, for providing fungal material.

\section{REFERENCES}

Abadio AK, Lima SS, Santana MF, Salomao TM, et al. (2012). Genetic diversity analysis of isolates of the fungal bean pathogen Pseudocercospora griseola from central and southern Brazil. Genet. Mol. Res. 11: 1272-1279.

Adinolfi B, Chicca A, Martinotti E, Breschi MC, et al. (2007). Sequence characterized amplified region (SCAR) analysis on DNA from the three medicinal Echinacea species. Fitoterapia 78: 43-45.

Altschul SF, Madden TL, Schaffer AA, Zhang J, et al. (1997). Gapped BLAST and PSI-BLAST: a new generation of protein database search programs. Nucleic Acids Res. 25: 3389-3402.

Arici SE and Koç NK (2010). RAPD-PCR analysis of genetic variation among isolates of Fusarium graminearum and Fusarium culmorum from wheat in Adana Turkey. Pak. J. Biol. Sci. 13: 138-142.

Bentley AR, Tunali B, Nicol JM, Burgess LW, et al. (2006). A survey of Fusarium species associated with wheat and grass stem bases in Northern Turkey. Sydowia 58: 163-177.

Carter JP, Rezanoor HN, Desjardins AE and Nicholson P (2000). Variation in Fusarium graminearum isolates from Nepal associated with their host of origin. Plant Pathol. 49: 452-460.

Carter JP, Rezanoor HN, Holden D, Desjardins AE, et al. (2002). Variation in pathogenicity associated with the genetic diversity of Fusarium graminearum. Eur. J. Plant Pathol. 108: 573-583.

Champail A, Dore T and Fourbet JF (2004). Fusarium head blight: epidemiological origin of the effects of cultural practices on head blight attack and the production of mycotoxins by Fusarium in wheat grains. Plant Sci. 166: 1389-1415.

Demir K, Bakir M, Sarikamis G and Acunalp S (2010). Genetic diversity of eggplant (Solanum melongena) germplasm from Turkey assessed by SSR and RAPD markers. Genet. Mol. Res. 9: 1568-1576.

FAO (2012). Food and Agriculture Organization of the United Nations (FAO). FAOSTAT, Italy. Avaible at [http://faostat. 
fao.org]. Accessed June 21, 2012.

Gutleb AC, Morrison E and Murk AJ (2002). Cytotoxicity assays for mycotoxins produced by Fusarium strains: a review. Environ Toxicol. Pharmacol. 11: 309-320.

Gürel F, Albayrak G, Diken O, Cepni E, et al. (2010). Use of REP-PCR for genetic diversity analyses in Fusarium culmorum. J. Phytopathol. 158: 387-389.

Haratian M, Sharifnabi B, Alizadeh A and Safaie N (2008). PCR analysis of the Tri13 gene to determine the genetic potential of Fusarium graminearum isolates from Iran to produce nivalenol and deoxynivalenol. Mycopathologia 166: 109-116.

Harlan JR and Zohary D (1966). Distribution of wild wheats and barley. Science 153: 1074-1080.

Heng MH, Baharuddin S and Latiffah Z (2012). Characterization of Fusarium species section Liseola by restriction analysis of the IGS region. Genet. Mol. Res. 11: 383-392.

Hue FX, Huerre M, Rouffault MA and de BC (1999). Specific detection of Fusarium species in blood and tissues by a PCR technique. J. Clin. Microbiol. 37: 2434-2438.

Mamluk OF, Cetin L, Braun HJ, Bolat N, et al. (1997). Current status of wheat and barley diseases in the Central Anatolian Planteau of Turkey. Phytopathol. Mediterr. 36: 167-181.

Memati Z, Tehranifar A, Farsi M, Mirshamsikakhki A, et al. (2012). Evaluation of genetic diversity of iranian pomegranate cultivars using fruit morphological characteristics and AFLP markers. Not. Bot. Horti. Agrobo. 40: 261-268.

Mesterházy A (1995). Types and components of resistance to Fusarium head blight of wheat. Plant Breed. 114: 377-386.

Miedaner T, Schilling AG and Geiger HH (2001). Molecular genetic diversity and variation for aggressiveness in populations of Fusarium graminearum and Fusarium culmorum sampled from wheat fields in different countries. $J$. Phytopathol. 149: 641-648.

Miedaner T, Cumagun CJR and Chakraborty S (2008). Population genetics of three important head blight pathogens Fusarium graminearum, F. pseudograminearum and F. culmorum. J. Phytopathol. 156: 129-139.

Mishra PK, Fox RTV and Culham A (2003). Inter-simple sequence repeat and aggressiveness analyses revealed high genetic diversity, recombination and long-range dispersal in Fusarium culmorum. Ann. Appl. Biol. 143: 291-301.

NCBI (2012). National Center for Biotechnology Information. Avaible at [http://www.ncbi.nlm.nih.gov/ nuccore/374258598]. Accessed June 22, 2012.

Nei M and Li WH (1979). Mathematical model for studying genetic variation in terms of restriction endonucleases. Proc. Natl. Acad. Sci. U. S. A. 76: 5269-5273.

Nicholson P, Simpson DR, Weston G, Rezanoor HN, et al. (1998). Detection and quantification of Fusarium culmorum and Fusarium graminearum in cereals using PCR assays. Physiol. Mol. Plant P. 53: 17-37.

Pandolfi V, Jorge EC, Melo CM, Albuquerque AC, et al. (2010). Gene expression profile of the plant pathogen Fusarium graminearum under the antagonistic effect of Pantoea agglomerans. Genet. Mol. Res. 9: 1298-1311.

Quellet T and Seifert KA (1993). Genetic characterization of Fusarium graminearum strains using RAPD and PCR amplification. Phytopathology 83: 1003-1007.

Saharan MS, Kumar J, Sharma AK and Nagarajan S (2004). Fusarium head blight (FHB) or head scab of wheat-A review. Proc. Indian Natl. Sci. Acad. 3: 255-268.

Saharan MS, Naef A, Kumar J and Tiwari R (2007). Characterization of variability among isolates of Fusarium graminearum associated with head scab of wheat using DNA markers. Curr. Sci. India 92: 230-235.

Schilling AG, Möller EM and Geiger HH (1996). Polymerase chain reaction-based assays for species-specific detection of Fusarium culmorum, F. graminearum and F. aveneceaum. Mol. Plant Pathol. 86: 515-522.

Tunali B, Nicol J, Erol FY and Altiparmak G (2006a). Pathogenicity of Turkish crown and head scab isolates on stem bases on winter wheat under greenhouse conditions. Plant Pathol. J. 5: 143-149.

Tunali B, Ozseven İ, Buyuk O, Erdurmus D, et al. (2006b). Fusarium head blight and deoxynivalenol accumulation of wheat in Marmara region and reactions of wheat cultivars and lines to F. graminearum and F. culmorum. Plant Pathol. J. 5: 150-156.

Virdi JS and Sachdeva P (2005). Genetic diversity of pathogenic microorganisms: basic insights, public health implications and the Indian initiatives. Curr. Sci. India 89: 113-123.

Walker SL, Leath S, Hagler WM and Murphy JP (2001). Variation among isolates of Fusarium graminearum associated with fusarium head blight in North Carolina. Plant Dis. 85: 404-410.

Ward E, Foster SJ, Fraaije BA and McCartney HA (2004). Plant pathogen diagnostics: immunological and nucleic acidbased approaches. Ann. Appl. Biol. 145: 1-16.

Yörük E and Albayrak G (2012). Chemotyping of Fusarium graminearum and F. culmorum isolates from Turkey by PCR assay. Mycopathologia 173: 53-61. 